\title{
Classification Schema of Symptomatic Enterogastric Reflux Utilizing Sincalide Augmentation on Hepatobiliary Scintigraphy
}

\author{
Matthew F. Covington ${ }^{1}$, Elizabeth Krupinski ${ }^{1}$, Ryan J. Avery ${ }^{1}$, and Phillip H. Kuo ${ }^{2}$ \\ ${ }^{I}$ Department of Medical Imaging, University of Arizona College of Medicine, Tucson, Arizona; and ${ }^{2}$ Department of Medical Imaging, \\ Medicine and Biomedical Engineering, University of Arizona College of Medicine, Tucson, Arizona
}

\begin{abstract}
Enterogastric reflux (EGR) is the reflux of duodenal contents into the stomach. Hepatobiliary scintigraphy provides physiologic assessment of the biliary system and was used to test the hypothesis that presence and timing of EGR may be associated with infusion of sincalide, a surrogate of endogenous cholecystokinin. Methods: One hundred fifty-seven hepatobiliary scintigraphy studies were retrospectively reviewed. Data included EGR incidence on initial reports, incidence after masked second reads, and time of EGR onset in relation to sincalide infusion. EGR cases were then classified according to onset on pre-, post-, or both presincalide and postsincalide imaging. Results: Time of EGR onset at 19-24 minutes after start of a 15-min sincalide infusion differed significantly from normal $(p<0.0001)$. EGR was initially reported in 14 of 157 cases $(8.9 \%)$ but found in 38 of 157 cases on masked second reads (24.2\%), corresponding to a $15.3 \%$ discrepancy rate. Conclusion: The temporal association of EGR onset with sincalide infusion may identify patients with EGR mimicking chronic cholecystitis or biliary dyskinesia. A novel classification schema was therefore developed as a framework for future research, utilizing EGR onset in relation to pre-, post-, or both presincalide and postsincalide imaging as a hypothetical biomarker of clinically significant EGR.
\end{abstract}

Key Words: enterogastric reflux; bile reflux; hepatobiliary scintigraphy; HIDA scan; sincalide

J Nucl Med Technol 2014; 42:198-202

DOI: 10.2967/jnmt.114.141168

$\mathbf{E}$ nterogastric reflux (EGR) is the reflux of bile and other digestive fluids from the duodenum into the stomach. EGR is a common but underdiagnosed entity that exerts harmful effects on gastric mucosa, facilitates Helicobacter pylori colonization, and acts synergistically with $\mathrm{H}$. pylori to cause chronic gastritis and intestinal metaplasia $(1,2)$. Duodenogastroesophageal reflux, wherein reflux extends from the duodenum into the esophagus, is associated with refractory

Received Apr. 4, 2014; revision accepted Jun. 23, 2014.

For correspondence or reprints contact: Phillip H. Kuo, 1501 N. Campbell

Ave., P.O. Box 245067, Tucson, AZ 85724-5067.

E-mail: philliphkuo@gmail.com

Published online Jul. 17, 2014.

COPYRIGHT (C 2014 by the Society of Nuclear Medicine and Molecular Imaging, Inc. gastroesophageal reflux disease and contributes to the development of Barrett esophagus and esophageal adenocarcinoma (3-5). Risk factors for EGR include gastric surgery and cholecystectomy $(2,6)$.

EGR may be the culprit for abdominal pain attributed to other common etiologies including cholecystitis (7). Options for diagnosing EGR include hepatobiliary scintigraphy scans, gastrointestinal endoscopy, direct gastric aspirate sampling, and automated measurements of bilirubin absorbance $(1,3,8,9)$. Multiple studies suggest hepatobiliary scintigraphy scans may be superior to other modalities for the detection of EGR $(10,11)$. However, review of the literature revealed a dearth of studies on the relationship between EGR and administration of sincalide, a surrogate of endogenous cholecystokinin. Sincalide elicits contraction of the gallbladder and relaxation of the sphincter of Oddi, therefore facilitating excretion of bile acids into the bowel. An altered physiologic response to sincalide, and thus also endogenous cholecystokinin, may potentially cause or augment EGR.

We performed a 12-mo retrospective review of all hepatobiliary scintigraphy scans to assess the correlation between the presence and timing of EGR in relation to sincalide. We hypothesized that sincalide, as an analog for endogenous cholecystokinin, may cause or exacerbate EGR and therefore replicate postprandial EGR. After a relationship between EGR and sincalide infusion was observed, a novel classification schema for EGR based on the presence of EGR on pre- and postsincalide hepatobiliary scintigraphy was developed as a hypothetical imaging biomarker for symptomatic EGR.

\section{MATERIALS AND METHODS}

Institutional Review Board approval was obtained before a retrospective review of the reports of all 157 hepatobiliary scintigraphy studies performed on adults at our institution from January 1, 2012, to December 31, 2012, and the requirement for informed consent was waived.

\section{Patient Population}

Inclusion criteria included all patients $18 \mathrm{y}$ or older who were referred for outpatient hepatobiliary scintigraphy to evaluate suspected chronic cholecystitis or biliary dyskinesia. Exclusion criteria 


\begin{tabular}{|c|c|c|c|}
\hline Class & Description & Incidence & $\begin{array}{l}\text { Subclassification of } \\
\text { persistent or transient }\end{array}$ \\
\hline \multirow[t]{2}{*}{ A } & $\begin{array}{l}\text { EGR on presincalide imaging with either no EGR on postsincalide } \\
\text { imaging or EGR on postsincalide imaging of the same or less } \\
\text { intensity than presincalide EGR. }\end{array}$ & 6 of 34 patients & 5 of 6 persistent \\
\hline & & & 1 of 6 transient \\
\hline B & EGR on postsincalide imaging only. & 21 of 34 patients & $\begin{array}{l}20 \text { of } 21 \text { persistent } \\
1 \text { of } 21 \text { transient }\end{array}$ \\
\hline \multirow[t]{2}{*}{ C } & $\begin{array}{l}\text { EGR on both presincalide and postsincalide imaging with increase in } \\
\text { EGR intensity during the postsincalide period. }\end{array}$ & 7 of 34 patients & 3 of 7 persistent \\
\hline & & & 4 of 7 transient \\
\hline
\end{tabular}

included minors, patients who were admitted to the hospital, or patients who were evaluated for acute cholecystitis.

\section{Outcome Measures}

Primary outcome measures included the presence or absence of EGR on initial hepatobiliary scintigraphy reports and on subsequent masked review. Time of EGR onset in minutes before or after sincalide infusion, if applicable, was also assessed. Secondary outcome measures included relationship of EGR to patient age and sex, megabecquerels $(\mathrm{mCi})$ of injected radiotracer, and gallbladder ejection fraction (GBEF), if performed.

To achieve the outcome measures, all studies were reevaluated independently by 2 board-certified radiologists with fellowship training in nuclear medicine, also the initial readers, for the presence and duration of EGR and timing of EGR pre- or postsincalide injection. Cases in which there was disagreement on the presence of EGR were then reviewed by both physicians together, and a consensus decision on the presence and duration of EGR was reached. For a consensus read to be positive for EGR, both readers needed to have a greater than $95 \%$ subjective confidence level for the presence of EGR. The readers were masked to the initial imaging reports during review.

EGR identification was evaluated using 3 criteria, all of which must have been present for a positive scan. These criteria consisted of location of radiotracer activity in the left upper quadrant, shape of activity with the expected configuration of the stomach, and activity that appeared distinct from small or large bowel (i.e., nonenteric activity).
On review, the readers also documented time of EGR onset as presincalide, postsincalide, or both presincalide and postsincalide according to EGR onset within each respective imaging period. The duration of EGR in minutes for each time period was also tabulated.

\section{Development of Classification Schema}

After masked review, every case of EGR was classified according to a novel classification schema, developed by the authors as a hypothetic biomarker and framework for future research on clinically symptomatic EGR (Table 1). Class A applies to cases in which EGR occurs only during presincalide imaging or EGR occurs during both presincalide and postsincalide imaging, with postsincalide EGR demonstrating equal or less intensity, compared with presincalide EGR (i.e., activity in the stomach does not increase after sincalide infusion) (Fig. 1). Class B applies to cases of EGR that occur exclusively during postsincalide imaging (Fig. 2). Class $\mathrm{C}$ applies to cases of EGR occurring during both presincalide and postsincalide imaging wherein EGR intensity increased during the postsincalide period (i.e., activity in the stomach increases after sincalide infusion) (Fig. 3). Each case of EGR was subclassified as either transient or persistent, meaning EGR was transient and resolved before completion of each respective imaging period or EGR persisted throughout the remainder of each respective imaging period (pre- or postsincalide).

\section{Imaging Technique}

Before imaging, all patients fasted for at least $6 \mathrm{~h}$. The technical parameters for hepatobiliary scintigraphy scan acquisition in-

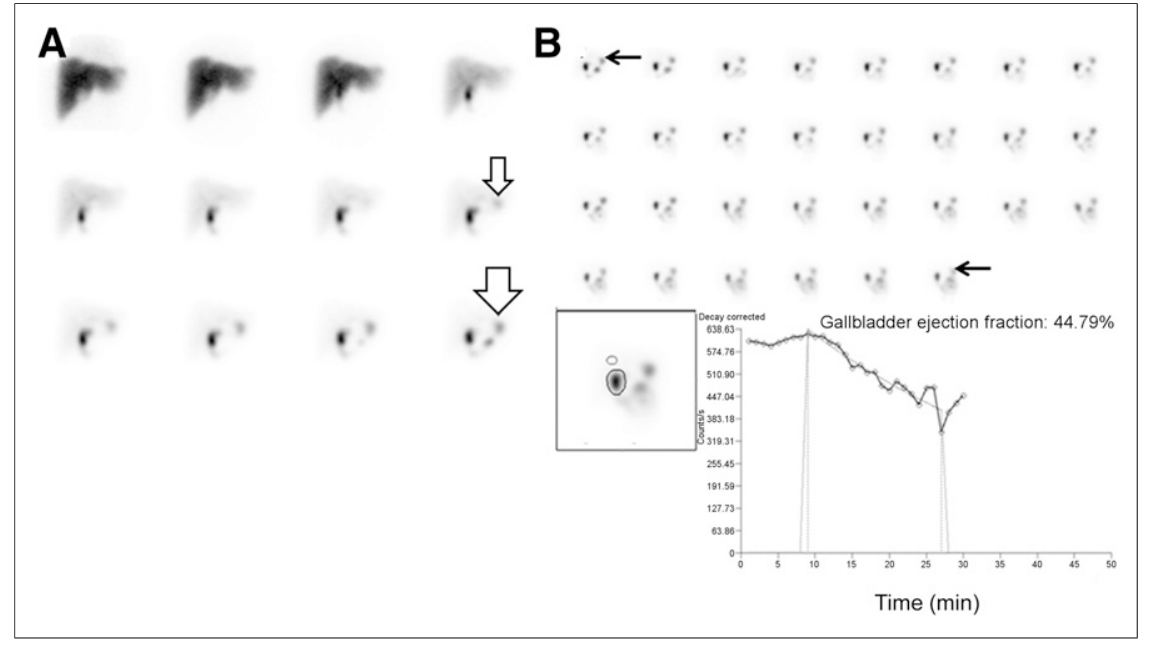

FIGURE 1. A 20-y-old woman with persistent abdominal pain. All images are presented in 5-min time frames. Example of class-A EGR: EGR is present on presincalide imaging with no increase in EGR intensity on postsincalide imaging. Presincalide images (A) show onset of EGR at $40 \mathrm{~min}$ (small open arrow) that persists throughout remainder of presincalide imaging (large open arrow). Postsincalide images (B) show EGR persisting throughout postsincalide imaging (black arrows), with no increase in intensity compared with presincalide EGR (A). Postsincalide imaging begins simultaneously with start of sincalide infusion. 
FIGURE 2. A 60-y-old man with chronic right upper quadrant pain. All images are presented in 5-min time frames. Example of class-B EGR: EGR is present on postsincalide images only. Presincalide images show no EGR (A). Postsincalide images (B) show EGR onset at $20 \mathrm{~min}$, which persists throughout remainder of imaging period (black arrows). Postsincalide imaging begins simultaneously with start of sincalide infusion. Presincalide imaging was stopped early at 50 min because patient left for restroom.

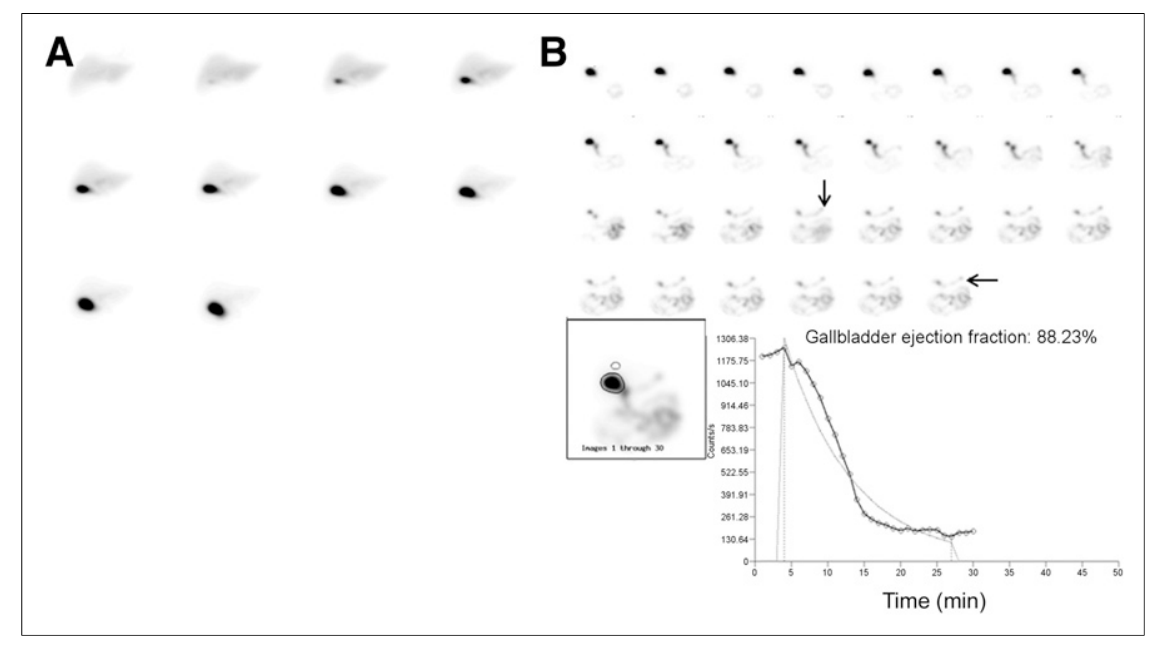

cluded anterior planar imaging presented as 1-, 3-, or 5-min frames, depending on the camera system used (either Axis [Philips] or Transcam [ADAC]), after the injection of an average of 192.4 MBq (5.2 mCi [range, 148-222 MBq, and 4.0-6.0 mCi]) of ${ }^{99 \mathrm{~m}} \mathrm{Tc}$-mebrofenin. GBEF was assessed with a 15 -min infusion using an injection pump of sincalide $(0.01 \mathrm{mcg} / \mathrm{kg}$ ) (Bracco Diagnostics, Inc.), followed by an additional $15 \mathrm{~min}$ of imaging. Depending on the camera system, the $30 \mathrm{~min}$ of imaging during and after infusion of sincalide was also presented as 1-, 3-, or 5min frames. To replicate the environment of the initial image interpretation, all images were reviewed in their original format without additional reprocessing.

\section{Statistical Methods}

A $\chi^{2}$ test was used to assess differences between sex and presence of EGR. Unpaired $t$ tests were used to assess differences between EGR as a function of patient age, concentration of injected radiotracer, and GBEF. A $z$ test was used to calculate the skew of EGR onset after sincalide administration. An ANOVA test was used to evaluate time of onset of bowel or gallbladder activity for cases with and without EGR. Significance was calculated as a $P$ value of less than or equal to 0.05 .

\section{RESULTS}

Retrospective review of original clinical reports revealed a reported EGR diagnosis in 14 of 157 cases, corresponding with an $8.9 \%$ reported EGR incidence in our population. Masked review of the clinical images revealed 40 cases of EGR diagnosed by reader 1 and 35 cases of EGR diagnosed by reader 2. Consensus between the readers revealed 38 cases of EGR corresponding with an EGR incidence of $24 \%$ (38/157 cases). Only 1 case of EGR diagnosed in the initial report was overturned on review. The remaining 13 cases of EGR diagnosed on initial reports were confirmed.

A $z$ test for the skew of EGR onset for 5-min intervals after sincalide administration revealed that the time of onset at 19-24 min after sincalide administration differed significantly from a statistically normal gaussian distribution $(P<$ 0.0001) (Fig. 4). This postsincalide result markedly differed from presincalide findings. A $z$ test of the skew of time of EGR onset on presincalide imaging revealed no significant deviation from a normal gaussian distribution.

GBEF was assessed in 117 of 157 cases, 43 of which were abnormal (ejection fraction $<35 \%$ ). After ${ }^{99 m} \mathrm{Tc}-$ mebrofenin injection, bowel activity was first seen at an average of $28 \mathrm{~min}$ for non-EGR cases and $34 \mathrm{~min}$ for EGR cases $(P=0.875)$. Gallbladder activity was observed at an average of 28 and 26 min for cases with and without EGR, respectively. An ANOVA test revealed no statistically significant difference for onset of bowel or gallbladder activity for cases

FIGURE 3. A 77-y-old woman with right upper quadrant pain. All images are presented in 5-min time frames. Example of class-C EGR: EGR is present on presincalide and postsincalide imaging, with increase in EGR intensity during postsincalide period. Presincalide images (A) demonstrate EGR that is definitively seen at 50 min (black arrow). Postsincalide images (B) show residual activity in stomach from presincalide imaging, with subsequent marked increase in activity at 18-24 min after initiation of sincalide

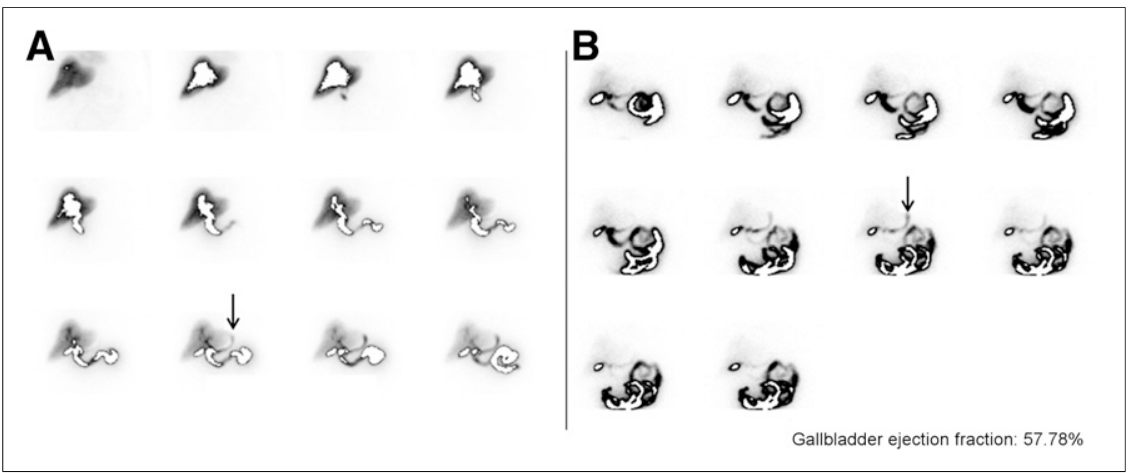
infusion consistent with additional EGR (black arrow). Therefore, this patient experienced EGR pre- and postsincalide infusion and is grouped into class $C$. Postsincalide imaging begins simultaneously with start of sincalide infusion. GBEF curve is not shown to allow better display of pre- and postsincalide images. 


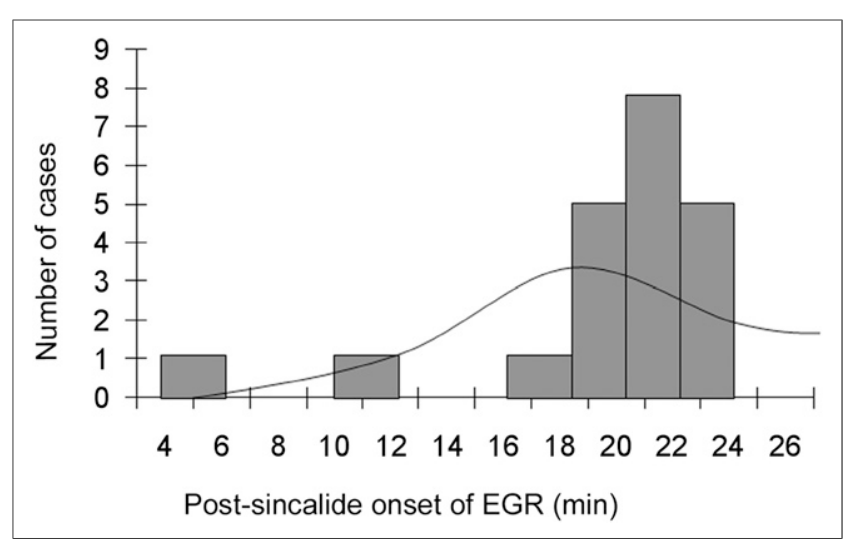

FIGURE 4. Time of EGR onset after sincalide infusion. Histogram plot and analysis of skew demonstrate that only time of EGR onset at 19-24 min after sincalide administration differed significantly from normal $(P<0.0001)$.

with and without EGR $(P=0.875)$ and revealed no significant interaction between these variables $(P=0.099)$.

The average patient age was 50.4 y (range, 18-90 y). Females comprised $68.2 \%$ of included patients (107/157 patients). A $\chi^{2}$ test demonstrated no statistically significant difference between genders, with EGR present in $26 \%$ of women and $20 \%$ of men $\left(\chi^{2}=0.707, P=0.401\right)$. Unpaired $t$ tests showed no statistically significant differences for EGR as a function of patient age $(P=0.724)$, activity of injected radiotracer $(P=0.788)$, and $\operatorname{GBEF}(P=0.996)$. There was no significant difference between EGR pre- or postsincalide as a function of age $(P=0.632)$, injected activity $(P=0.517)$, or GBEF $(P=0.851)$ (Table 2$)$.

It was not possible to classify 4 of the 38 cases of EGR because sincalide was not given during these examinations; therefore, 34 cases could be classified into our schema (Table 1). Class-A EGR was identified in 6 of 34 patients, 5 of which were persistent and 1 was transient. Class-B EGR was identified in 21 of 34 patients, with a persistent pattern in 20 cases and a transient pattern in a single case. Class-C EGR was identified in 7 of 34 patients, with a persistent pattern in 3 cases and a transient pattern in 4 cases.

\section{DISCUSSION}

The frequent onset of EGR during the interval of 1924 min after initiation of sincalide infusion was highly statistically significant $(P<0.0001)$. This recognition of when EGR is most likely to occur may increase a radiologist's sensitivity for detecting EGR. This finding also implies that sincalide infusion during hepatobiliary scintigraphy or during endoscopy may augment the sensitivity of EGR detection. Indeed, $62 \%$ of EGR cases in our study were manifest only on postsincalide imaging. Because sincalide infusion acts as a surrogate to endogenous release of cholecystokinin stimulated by eating, the relationship of EGR to sincalide in the prandial and postprandial period may improve understanding of the pathophysiology of EGR, suggesting a contributory role by cholecystokinin. Future research on the clinical relevance of this finding is needed.

The novel classification schema may identify cases of EGR most likely to mimic chronic cholecystitis or biliary dyskinesia. This identification is important because cholecystectomy is a known risk factor for worsening $\operatorname{EGR}(2,6)$. According to our model, class-A EGR patients would have clinical symptoms unrelated to eating and would be unlikely to have EGR that mimics gallbladder pain. Patients with class-B EGR would have clinical symptoms predominantly during meals or in the postprandial state, thereby mimicking cases of chronic cholecystitis or biliary dyskinesia. Patients with class-C EGR, wherein EGR worsens during the postsincalide period, may have a combination of symptoms unrelated to eating that worsen in the postprandial state and also may mimic gallbladder pathology. Patients subclassified with persistent EGR would expect overall worse severity and longer duration of pain, and patients with a subclassification of transient EGR may have more mild symptoms and intermittent pain. In our study, $82 \%$ of patients with EGR had either class-B or class-C EGR. The classification schema proposes a framework for future studies of other imaging biomarkers and clinical or endoscopic correlations.

At least 2 possibilities explain the onset of EGR during the interval of 19-24 min after initiation of sincalide infusion. The first is that EGR onset postsincalide may be a function of time from start of sincalide infusion, thereby occurring approximately 20 min after the initiation of the 15 -min sincalide infusion. The alternate explanation is that EGR onset is related to the wearing-off of sincalide, which has a half-life of $2.5 \mathrm{~min}$ (12). This 4-9 $\min$ (2-4 half-lives) after sincalide infusion would be the appropriate time frame for sincalide to wear off. Cholecystokinin and sincalide stimulate intestinal motility with concomitant contraction of

TABLE 2

Determination of Significance for Presence and Absence of EGR as Function of Patient Age, Sex, MBq (mCi) of Injected Activity, and GBEF

\begin{tabular}{lccc}
\hline \multicolumn{1}{c}{ Characteristic } & Reflux present $(n=38)$ & Reflux absent $(n=157)$ & Significance \\
\hline Average age $(\mathrm{y})$ & 51.3 & 50.2 & $P=0.724$ \\
Sex & $26 \%$ of females & $74 \%$ of females & $\chi^{2}=0.707, P=0.401$ \\
MBq $(\mathrm{mCi})$ injected activity & $20 \%$ of males & $80 \%$ of males & $P=0.788$ \\
GBEF $(n=117)$ & $192.77(5.21)$ & $193.88(5.24)$ & $P=0.996$ \\
\hline
\end{tabular}


the pylorus $(12,13)$. A hypothetical physiologic mechanism for EGR is that the effect on pyloric contraction is either incomplete or of shorter duration than increased small bowel motility, therefore prompting reflux into the stomach. The total dose and rate of administration of sincalide may also be important variables. In addition, unlike a 15-min intravenous infusion of sincalide, endogenous cholecystokinin release may last up to several hours after a meal (12). The longer duration of endogenous cholecystokinin release may increase the probability of EGR if pyloric contraction is insufficient given a longer duration of bile flow through the open sphincter of Oddi.

In addition to the limitations already discussed, it is unknown whether the patients in the study had prior gastrointestinal surgery or were on medications such as sucralfate or proton pump inhibitors, which are proposed EGR treatments (6). Future studies should correlate clinical history and findings on endoscopy with the proposed classification system.

We detected a $15.1 \%$ discrepancy rate between the incidence of EGR initially reported in our clinical practice $(8.9 \%)$ and that detected on subsequent masked review with directed diagnostic attention (24\%). Our revised EGR incidence approaches that in the peer-reviewed literature, ranging between $26 \%$ and $58 \%(7,14)$. EGR was underreported in our institution, and this may be representative of other practices as well. If so, this underreporting suggests an opportunity for further physician education.

\section{CONCLUSION}

Masked review of 157 consecutive hepatobiliary scans from routine clinical practice at our institution revealed that time of EGR onset was found to be highly statistically significant at 19-24 min after initiation of a 15-min sincalide infusion. Therefore, detection of EGR may be augmented by sincalide administration and most reliably identified in this time window. A novel classification scheme based on timing of EGR in relation to sincalide infusion may have utility for future research to predict clinical symptoms related to EGR and identify cases of EGR that mimic cholecystitis or biliary dyskinesia.

\section{DISCLOSURE}

No potential conflicts of interest were reported.

\section{REFERENCES}

1. Chen SL, Mo JZ, Cao ZJ, Chen XY, Xiao SD. Effects of bile reflux on gastric mucosal lesions in patients with dyspepsia or chronic gastritis. World J Gastroenterol. 2005;11:2834-2837.

2. Zullo A, Rinaldi V, Hassan C, Lauria V, Attilli AF. Gastric pathology in cholecystectomy patients: role of Helicobacter pylori and bile reflux. J Clin Gastroenterol. 1998;27:335-338.

3. Hak NG, Mostafa M, Salah T, et al. Acid and bile reflux in erosive reflux disease, non-erosive reflux disease and Barrett's esophagus. Hepatogastroenterology. 2008;55:442-447.

4. Jürgens S, Meyer F, Spechler SJ, Souza R. The role of bile acids in the neoplastic progression of Barrett's esophagus: a short representative overview. Z Gastroenterol. 2012;50:1028-1034.

5. Miyashita T, Miwa K, Fujimara T, et al. The severity of duodeno-esophageal reflux influences the development of different histological types of esophageal cancer in a rat model. Int J Cancer. 2013;132:1496-1504.

6. Madura JA. Primary bile reflux gastritis: diagnosis and surgical treatment. Am J Surg. 2003;186:269-273.

7. Arroyo AJ, Burns JB, Huyghe WA, Dollman AE, Patel YP. Enterogastric reflux mimicking gallbladder disease: detection, quantitation and potential significance. J Nucl Med Technol. 1999;27:207-214.

8. Houghton PW, Mortensen NJ, Thomas WE, et al. Intragastric bile acids and scintigraphy in the assessment of duodenogastric reflux. Br J Surg. 1986;73:292-294.

9. Padhy AK, Losu V, Shukla NK, et al. Thoracic stomach: comparative evaluation of endoscopy, gastric aspirate analysis and hepatobiliary scintigraphy in the diagnosis of duodeno-gastric reflux. Indian J Gastroenterol. 1990;9:277-279.

10. Zoras O, Chrysos E, Tzovaras G, et al. A composite score of enterogastric reflux quantitation on ${ }^{99 \mathrm{~m}} \mathrm{Tc}$-hepatobiliary scintigraphy. Hepatogastroenterology. 1995; 42:847-850.

11. Mittal BR, Ibrarullah M, Agarwal DK, et al. Comparative evaluation of scintigraphy and upper gastrointestinal tract endoscopy for detection of duodenogastric reflux. Ann Nucl Med. 1994;8:183-186.

12. Krishnamurthy GT, Krishnamurthy S. Gallbladder, sphincter of Oddi, cholecystokinin, and opioid interrelationship: effect of cholecystokinin on the gallbladder and sphincter of oddi. In: Nuclear Hepatology: A Textbook of Hepatobiliary Diseases. New York, NY: Springer Publishing; 2000:129-136.

13. Kinevac [package insert]. Princeton, NJ: Bracco Diagnostic, Inc.; May 2002.

14. Colletti PM, Barakos JA, Siegel ME, Ralls ME, Halls JM. Enterogastric reflux in suspected acute cholecystitis. Clin Nucl Med. 1987;12:533-535. 